\title{
A Novel Conformal RFID-Enabled Module Utilizing Inkjet-Printed Antennas and Carbon Nanotubes for Gas-Detection Applications
}

\author{
Li Yang, Student Member, IEEE, Rongwei Zhang, Student Member, IEEE, Daniela Staiculescu, Member, IEEE, \\ C. P. Wong, Fellow, IEEE, and Manos M. Tentzeris, Senior Member, IEEE
}

\begin{abstract}
This letter introduces for the first time the integration of a conformal radio frequency identification (RFID) antenna with a single-walled carbon nanotube (SWCNT) composite in a chipless RFID node for toxic gas detection. The electrical performance characterization of the inkjet-printed SWCNT film is also reported for the first time up to $1 \mathrm{GHz}$. The whole module is realized by inkjet printing on a low-cost paper-based substrate, and the RFID tag is designed for the European UHF RFID band. The electrical conductivity of the SWCNT film changes in the presence of very small quantities of toxic gases like ammonia and nitrogen oxide, resulting in the variation of the backscattered power level, which can be easily detected by the RFID reader to realize reliable wireless toxic gas sensing.
\end{abstract}

Index Terms-Carbon nanotube (CNT) composites, conformal antennas, gas sensing, inkjet printing, radio frequency identification (RFID), wireless sensor.

\section{INTRODUCTION}

$\mathbf{T}$ HE steadily growing radio frequency identification (RFID) industry requires an ever improving performance of the RFID systems, including reduced size and cost, and higher levels of integration. Also, the tag flexibility is becoming a must for almost all applications, including body area networks for medical systems, tracking for pharmaceutical and food industries, supply chain, space, and many more. This demand is further enhanced by the need for lightweight, reliable, and durable wireless RFID-enabled sensor nodes [1]. Hence, the two major challenges for such applications are the choice of the material and the advanced integration capabilities. The choice of paper as the substrate material presents multiple advantages and has established paper as one of the most promising materials for UHF RFID applications: The mass "reel-to-reel" production makes it the cheapest material ever made. Previous work has demonstrated the successful development of a fully inkjet-printed RFID module on paper [2]. The next challenge is to integrate the sensor on the paper substrate as well. Some passive RFID prototypes for sensing applications have been proposed [3], [4]. However, the sensing capabilities are usually realized by adding a discrete sensor or a special coating to the

Manuscript received February 19, 2009; revised March 29, 2009 and May 11, 2009. First published May 29, 2009; current version published July 09, 2009.

The authors are with the Georgia Institute of Technology, Atlanta, GA 30332 USA (e-mail: liyang@ece.gatech.edu).

Color versions of one or more of the figures in this letter are available online at http://ieeexplore.ieee.org.

Digital Object Identifier 10.1109/LAWP.2009.2024104
RFID tag, resulting in the difficulty in low-profile integration. Plus, the sensitivity is usually low. Therefore, there has been a growing interest in looking for new materials in RFID sensing applications: an ultra sensitive composite that can be printed directly on the same paper together with the antenna, for a low cost, flexible, highly integrated RFID module.

Carbon nanotubes (CNT) composites have been found to have electrical conductance highly sensitive to extremely small quantities of gases, such as ammonia $\left(\mathrm{NH}_{3}\right)$ and nitrogen oxide $\left(\mathrm{NO}_{x}\right)$ [5], and be compatible with inkjet printing [6]. However, due to the insufficient molecular network formation among the inkjet-printed CNT particles at nanoscale, instabilities were observed in both the resistance and, especially, the reactance dependence on frequency above several $\mathrm{MHz}$, which limits the CNT application in only dc or LF band [7]. To allow the CNT-enabled sensor to be integrated with RFID antenna at UHF band, a special recipe needs to be developed.

This letter presents, for the first time, a conformal CNT-based RFID-enabled sensor node for gas-sensing applications, fully printed directly on paper substrate. Specifically, in this study, one benchmarking RFID tag was designed for the European UHF RFID band centering at $868 \mathrm{MHz}$. The printed CNT particles were single-walled carbon nanotubes (SWCNT) from Carbon Solutions, which were dispersed in dimethylformamide (DMF) solution and sonicated to meet the viscosity requirement for the inkjet printer. The impedance of the SWCNT film forms the sensor part. The antenna was printed first, followed by the 25 layers of the dispersed SWCNT as a load with "gas-controlled" value. When $4 \%$ consistency ammonia was imported into the gas chamber, the SWCNT impedance changed from $51.6-j 6.1 \Omega$ to $97.1-j 18.8 \Omega$ at $868 \mathrm{MHz}$, resulting in a 10.8-dBi variation in the backscattered power from the tag antenna, that can be easily detected by the RFID reader to realize the "real-time" gas detection.

\section{INKJET-PRINTED SWCNT}

As a direct-write technology, inkjet printing transfers the pattern directly to the substrate. Due to its capability of jetting one single ink droplet in the amount as low as $1 \mathrm{pL}$, it has widely drawn attention from the industrial world as a more accurate and economic fabrication method than the traditional lithography method.

To enable the SWCNT to be inkjet-printed, a SWCNT ink solution was developed as the first step. Two types of SWCNT-namely, P2-SWNT and P3-SWNT-were tested. 


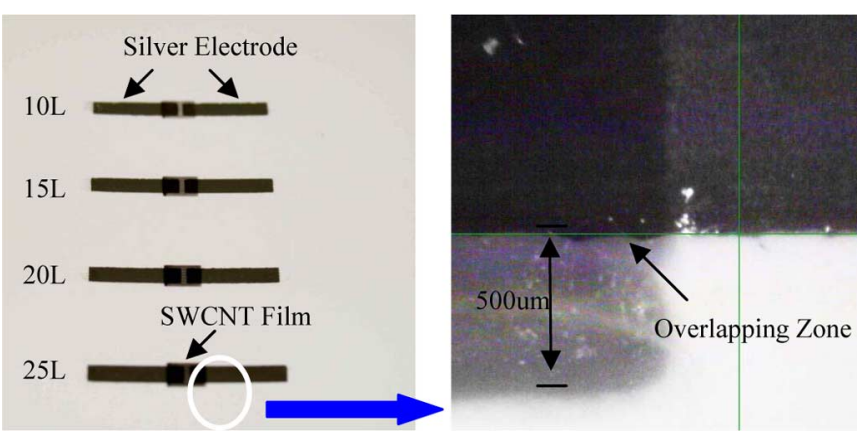

Fig. 1. Photograph of the inkjet-printed SWCNT films with silver electrodes. The SWCNT layers of the samples from top to bottom are 10, 15, 20, and 25, respectively. The dark region in the magnified picture shows the overlapping zone between the SWCNT film and the silver electrodes.

P2-SWNT is developed from purified AP-SWNT by air oxidation and catalyst removing. P3-SWNT is developed from AP-SWNT purified with nitric acid. Compared with P2-SWNT, P3-SWNT has much higher functionality and is easier to disperse in the solvent. In experiments, P2-SWNT started to aggregate at the concentration lower than $0.1 \mathrm{mg} / \mathrm{mL}$, while P3-SWNT can go up to $0.4 \mathrm{mg} / \mathrm{mL}$ and still show good dispersion. Therefore, P3-SWNT was selected for latter steps.

The sample SWCNT powder was dispersed in DMF, a polar aprotic solvent. The concentration of the ink was $0.4 \mathrm{mg} / \mathrm{mL}$. This high concentration helped the nanoparticle network formation after printing; otherwise, there would be instability in the impedance response versus frequency of the SWCNT film due to insufficient network formation, such as a sharp dropping of resistance value after $10 \mathrm{MHz}$ [4]. The diluted solution was purified by sonication for $12 \mathrm{~h}$ to prevent aggregations of large particle residues. This is important to avoid the nozzle clogging by SWCNT flocculation during the printing process. Dimatix DMCLCP-11610 printer head was used to eject the SWCNT ink droplet.

Silver electrodes were patterned with the nanopractical ink from Cabot before depositing the SWCNT film, followed by a $140^{\circ} \mathrm{C}$ sintering. The electrode finger is $2 \mathrm{~mm} \times 10 \mathrm{~mm}$ with a gap of $0.8 \mathrm{~mm}$. Then, the $3 \mathrm{~mm} \times 2 \mathrm{~mm}$ SWCNT film was deposited. The $0.6-\mathrm{mm}$ overlapping zone is to ensure the good contact between the SWCNT film and the electrodes. Four devices with $10,15,20$, and 25 SWCNT were fabricated to investigate the electrical properties. Fig. 1 shows the fabricated samples.

CNT composites have an affinity for gas molecules. The absorption of gas molecules in the CNT changes the conductivity of the material, which can be explained by the charge transfer of reactive gas molecules with semiconducting CNT [5]. The electrical resistance of the fabricated device was measured by probing the end tips of the two electrodes. The dc results are shown in Fig. 2. The resistance goes down when the number of SWCNT layers increases. Since a high number of SWCNT overwritten layers will also help the nanoparticle network formation, 25-layer film is expected to have the most stable impedance-frequency response and is selected for the gas measurement. In the experiment, $4 \%$ consistency ammonia, which was widely used in chemistry plants, was guided into an 18-in tube-shape gas flowing chamber connected with an exhaust hood. The test

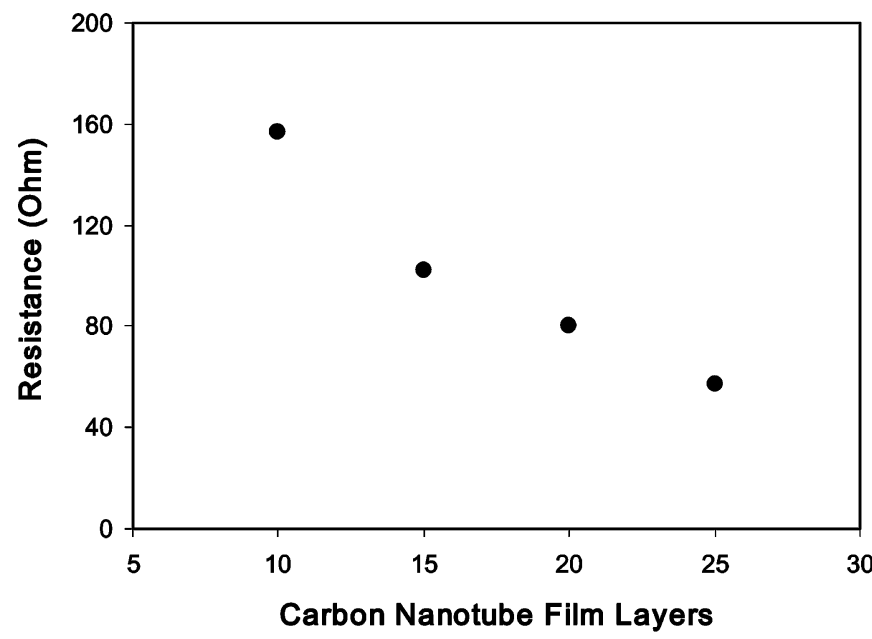

Fig. 2. Measured dc electrical resistance of SWCNT films.

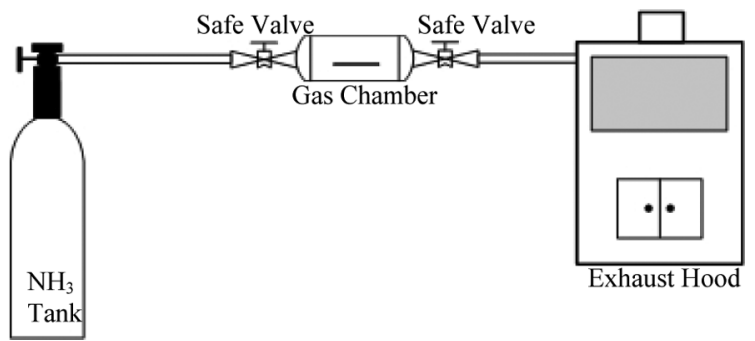

Fig. 3. Schematic of $\mathrm{NH}_{3}$ gas detection measurement.

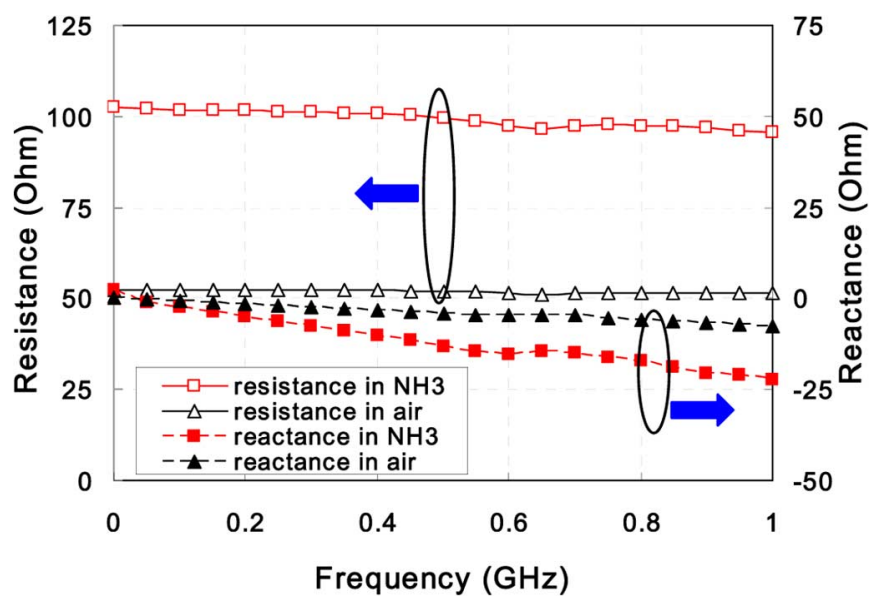

Fig. 4. Measured impedance characteristics of SWCNT film with 25 layers.

setup is shown in Fig. 3. The SWCNT film exhibits a fast-whilemonotonic impedance response curve to the gas flow [8]. A network vector analyzer (Rohde\&Schwarz ZVA8) was used to characterize the SWCNT film electrical performance at UHF band before and after the gas flowing. A GS probe was placed on the silver electrodes for the impedance measurements. The calibration method used was short-open-load-thru (SOLT). In Fig. 4, the gas sensor of SWCNT composite shows a very stable impedance response up to $1 \mathrm{GHz}$, which verifies the effectiveness of the developed SWCNT solvent recipe. At $868 \mathrm{MHz}$, the sensor exhibits a resistance of $51.6 \Omega$ and a reactance of $-j 6.1 \Omega$ 


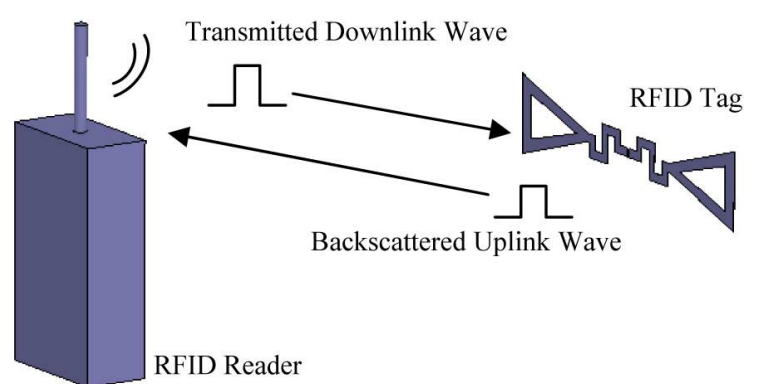

Fig. 5. Conceptual diagram of the proposed RFID-enabled sensor module.

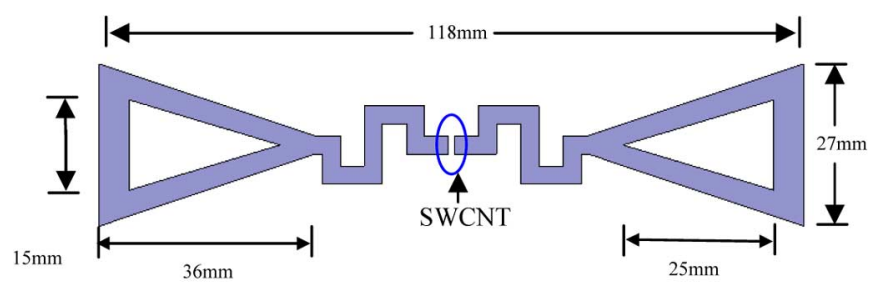

(a)

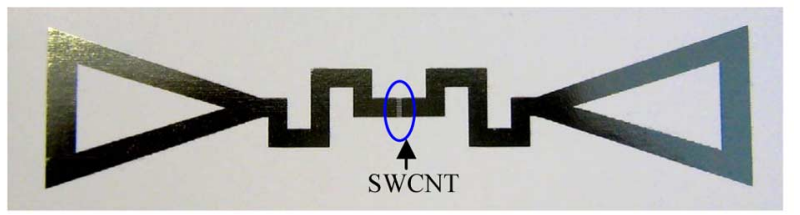

(b)

Fig. 6. The RFID tag module design on flexible substrate: (a) configuration; (b) photograph of the tag with inkjet-printed SWCNT film as a load.

in air. After meeting ammonia, the resistance was increased to $97.1 \Omega$, and the reactance was shifted to $-j 18.8 \Omega$.

\section{RFID TAG MODULE}

A passive RFID system operates in the following way. The RFID reader sends an interrogating RF signal to the RFID tag consisting of an antenna and an IC chip as a load. The IC responds to the reader by varying its input impedance, thus modulating the backscattered signal. The modulation scheme often used in RFID applications is amplitude shift keying (ASK), in which the IC impedance switches between the matched state and the mismatched state [9]. The power reflection coefficient of the RFID antenna can be calculated as a measure to evaluate the reflected wave strength.

$$
\eta=\left|\frac{Z_{\mathrm{Load}}-Z_{\mathrm{ANT}}^{*}}{Z_{\mathrm{Load}}+Z_{\mathrm{ANT}}}\right|^{2}
$$

where $Z_{\mathrm{Load}}$ represents the impedance of the load, and $Z_{\mathrm{ANT}}$ represents the impedance of the antenna terminals, with $Z_{\mathrm{ANT}}^{*}$ being its complex conjugate. The same mechanism can be used to realize RFID-enabled sensor modules. The SWCNT film functions as a tunable resistor $Z_{\mathrm{Load}}$ with a value determined by the existence of the target gas. The RFID reader monitors the backscattered power level. When the power level changes, it means that there is variation in the load impedance; therefore,

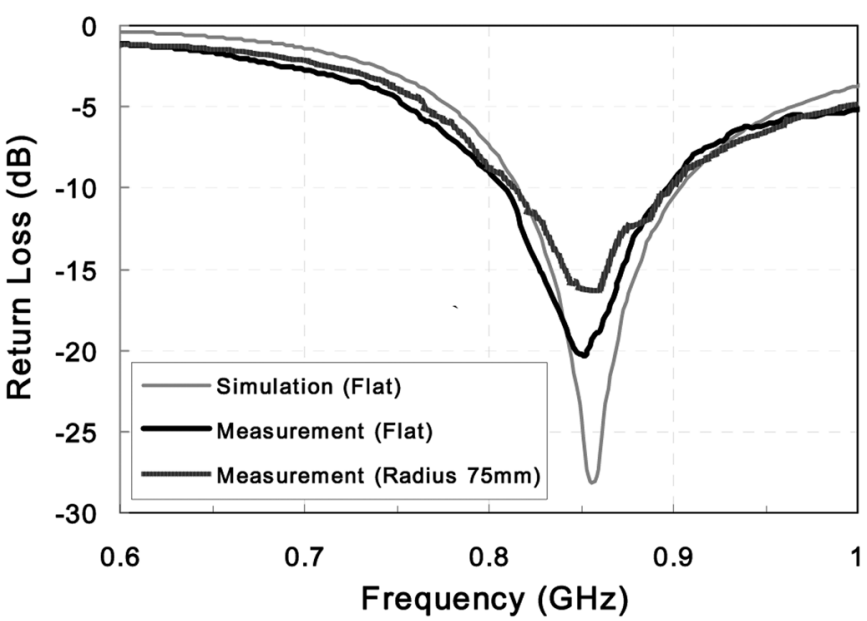

Fig. 7. Simulated and measured return loss of the RFID tag antenna.

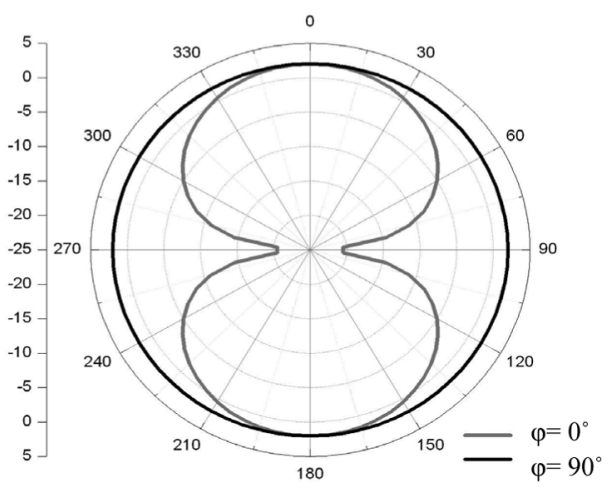

Fig. 8. Far-field radiation pattern plots.

the sensor detects the existence of the gas, as illustrated in Fig. 5.

The expected power levels of the received signal at the load of the RFID antenna can be calculated using Friis free-space formula, as

$$
P_{\mathrm{tag}}=P_{t} G_{t} G_{r}\left(\frac{\lambda}{4 \pi d}\right)^{2}
$$

where $P_{t}$ is the power fed into the reader antenna, $G_{t}$ and $G_{r}$ are the gain of the reader antenna and tag antenna, respectively, and $d$ is the distance between the reader and the tag.

Due to the mismatch between the SWCNT sensor and tag antenna, a portion of the received power would be reflected back, as

$$
P_{\text {ref }}=P_{\text {tag }} \eta
$$

where $\eta$ is the power reflection coefficient in (1). Hence, the backscattered power received by the RFID reader is defined as

$$
P_{r}=P_{\mathrm{ref}} G_{t} G_{r} \eta\left(\frac{\lambda}{4 \pi d}\right)^{2}=P_{t} G_{t}^{2} G_{r}^{2} \eta\left(\frac{\lambda}{4 \pi d}\right)^{4}
$$

or written in a decibel form, as

$$
P_{r}=P_{t}+2 G_{t}+2 G_{r}-40 \log _{10}\left(\frac{4 \pi}{\lambda}\right)-40 \log _{10}(d)+\eta
$$




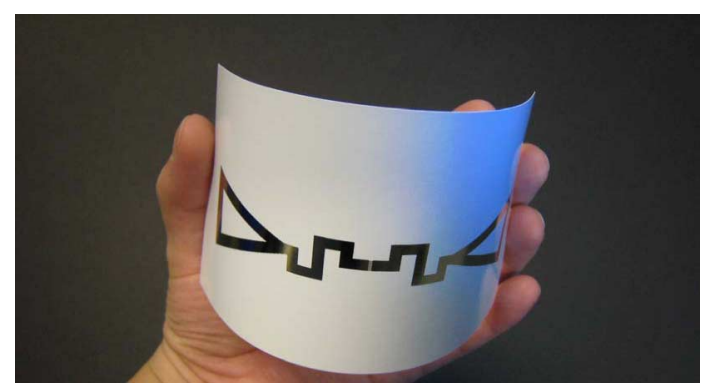

Fig. 9. Photograph of the conformal tag with a SWCNT film in the center.

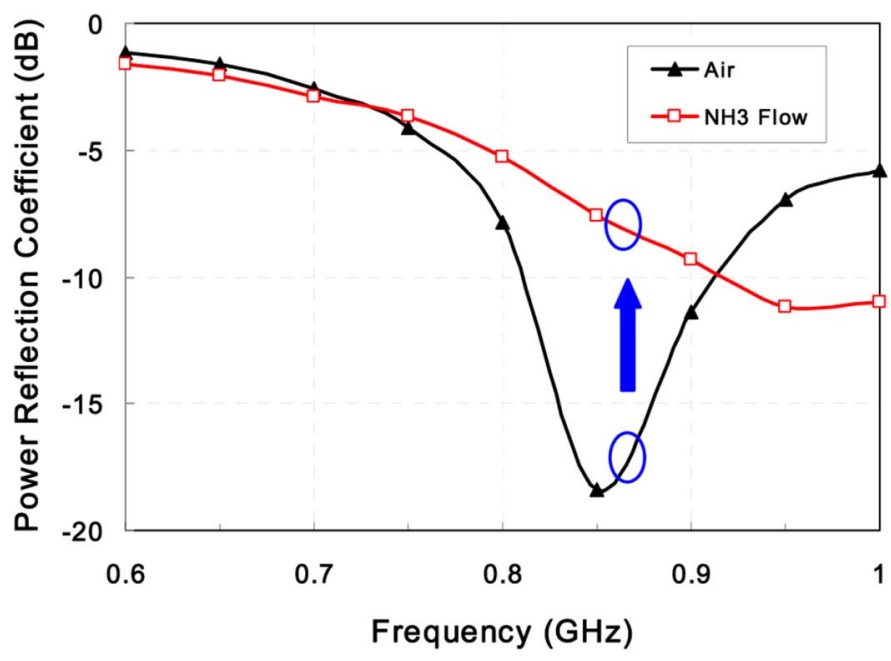

Fig. 10. The calculated power reflection coefficient of the RFID tag antenna with a SWCNT film before and after the gas flow.

where, except for the term of $\eta$, all the other values remain constant before and after the RFID tag meets gas. Therefore, the variation of the backscattered power level solely depends on $\eta$, which is determined by the impedance of the SWCNT film.

A bowtie meander-line dipole antenna was designed and fabricated on a $100-\mu \mathrm{m}$ thickness flexible paper substrate with dielectric constant 3.2. The RFID prototype structure is shown in Fig. 6 along with dimensions, with the SWCNT film inkjet printed in the center. The nature of the bowtie shape offers a more broadband operation for the dipole antenna.

A dielectric probe station was used for the impedance measurements. The measured $Z_{\mathrm{ANT}}$ at $868 \mathrm{MHz}$ is $42.6+j 11.4 \Omega$. The simulation and measurement results of the return loss of the proposed antenna are shown in Fig. 7, showing a good agreement. The tag bandwidth extends from 810 to $890 \mathrm{MHz}$, covering the whole European RFID band. The radiation pattern is plotted in Fig. 8, which is almost omnidirectional at $868 \mathrm{MHz}$ with directivity around $2.01 \mathrm{dBi}$ and $94.2 \%$ radiation efficiency. In order to verify the performance of the conformal antenna, measurements were performed as well by sticking the same tag on a 75-mm-radius foam cylinder. As shown in Fig. 7, there is almost no frequency shifting observed, with a bandwidth extending from 814 to $891 \mathrm{MHz}$. The directivity is slightly decreased to $1.99 \mathrm{dBi}$ with $90.3 \%$ radiation efficiency. Overall, a good performance still remains with the interested band covered. Fig. 9 shows a photograph of the designed conformal tag.

In air, the SWCNT film exhibited an impedance of $51.6-$ $j 6.1 \Omega$, which results in a power reflection at $-18.4 \mathrm{~dB}$. When $\mathrm{NH}_{3}$ is present, SWCNT film's impedance was shifted to $97.1-$ $j 18.8 \Omega$. The mismatch at the antenna port increased the power reflection to $-7.6 \mathrm{~dB}$. From (5), there would be $10.8-\mathrm{dBi}$ increase at the received backscattered power level, as shown in Fig. 10. By detecting this backscattered power difference on the reader's side, the sensing function can be fulfilled.

\section{CONCLUSION}

The inkjet printing method has been utilized for the first time to deposit SWCNT film on a fully printed UHF RFID module on paper to form a wireless gas sensor node. To ensure reliable inkjet printing, a SWCNT ink solution has been developed. The impedance performance of the SWCNT film was also characterized up to $1 \mathrm{GHz}$ for the first time. The design demonstrated the great applicability of inkjet-printed CNT for the realization of fully integrated "green" wireless RFID-enabled flexible sensor nodes based on the ultrasensitive variabiliity of the resistive properties of the CNT materials.

\section{REFERENCES}

[1] T. Mishima, N. Abe, K. Tanaka, and H. Taki, "Toward construction of a mobile system with long-range RFID sensors," in Proc. IEEE. Conf. Cybernetics Intell. Syst., 2004, vol. 2, pp. 960-965.

[2] L. Yang, A. Rida, R. Vyas, and M. M. Tentzeris, "RFID tag and RF structures on a paper substrate using inkjet-printing technology," IEEE Trans. Microw. Theory Tech., vol. 55, no. 12, pp. 2894-2901, Dec. 2007.

[3] M. Philipose, J. Smith, B. Jiang, A. Mamishev, and K. Sundara-Rajan, "Battery-free wireless identification and sensing," IEEE Pervasive Comput., vol. 4, no. 1, pp. 37-45, 2005.

[4] S. Johan, Z. Xuezhi, T. Unander, A. Koptyug, and H. Nilsson, "Remote moisture sensing utilizing ordinary RFID tags," in Proc. IEEE Sensors 2007, pp. 308-311.

[5] K. G. Ong and K. Zeng, "A wireless, passive carbon nanotube-based gas sensor,” IEEE Sensors J., vol. 2, no. 2, pp. 82-88, Apr. 2002.

[6] J. Song, J. Kim, Y. Yoon, B. Choi, and C. Han, "Inkjet printing of singe-walled carbon nanotubes and electrical characterization of the line pattern," Nanotechnol., vol. 19, no. 9, p. 095702, 2008.

[7] M. Dragoman, E. Flahaut, D. Dragoman, M. Ahmad, and R. Plana, "Writing electronic devices on paper with carbon nanotube ink," 2009, ArXiv-0901.0362.

[8] J. Yun, H. Chang-Soo, J. Kim, J. Song, and Y. Park, "Fabrication of carbon nanotube sensor device by inkjet printing," in Proc. IEEE Nano Eng. Molecular Syst. Conf., 2008, pp. 506-509.

[9] P. V. Nikitin and K. V. S. Rao, "Performance limitations of passive UHF RFID systems," in Proc. IEEE Symp. Antennas Propag., Jul. 2006, pp. 1011-1014. 